\title{
A magnetic resonance imaging (MRI)-based nomogram for predicting lymph node metastasis in rectal cancer: a node-for-node comparative study of MRI and histopathology
}

\author{
Yuan Liu ${ }^{1 \#}$, Lijuan Wan ${ }^{1 \#}$, Wenjing Peng ${ }^{1}$, Shuangmei Zou ${ }^{2}$, Zhaoxu Zheng ${ }^{3}$, Feng Ye ${ }^{1}$, Jun Jiang ${ }^{1}$, \\ Han Ouyang ${ }^{1}$, Xinming Zhao ${ }^{1}$, Hongmei Zhang ${ }^{1 \wedge}$
}

${ }^{1}$ Department of Radiology, National Cancer Center/National Clinical Research Center for Cancer/Cancer Hospital, Chinese Academy of Medical Sciences and Peking Union Medical College, Beijing, China; ${ }^{2}$ Department of Pathology, National Cancer Center/National Clinical Research Center for Cancer/Cancer Hospital, Chinese Academy of Medical Sciences and Peking Union Medical College, Beijing, China; ${ }^{3}$ Department of Colorectal Oncology, National Cancer Center/National Clinical Research Center for Cancer/Cancer Hospital, Chinese Academy of Medical Sciences and Peking Union Medical College, Beijing, China

\#These authors contributed equally to this work.

Correspondence to: Hongmei Zhang. Department of Radiology, National Cancer Center/National Clinical Research Center for Cancer/Cancer Hospital, Chinese Academy of Medical Sciences and Peking Union Medical College, \#17 Panjiayuan Nanli, Chaoyang District, Beijing 100021, China. Email: 13581968865@163.com.

Background: The aim of the present study was to investigate the potential risk factors for lymph node metastasis (LNM) in rectal cancer using magnetic resonance imaging (MRI), and to construct and validate a nomogram to predict its occurrence with node-for-node histopathological validation.

Methods: Our prediction model was developed between March 2015 and August 2016 using a prospective primary cohort (32 patients, mean age: 57.3 years) that included 324 lymph nodes (LNs) from MR images with node-for-node histopathological validation. We evaluated multiple MRI variables, and a multivariable logistic regression analysis was used to develop the predictive nomogram. The performance of the nomogram was assessed with respect to its calibration, discrimination, and clinical usefulness. The performance of the nomogram in predicting LNM was validated in an independent clinical validation cohort comprising 182 consecutive patients. Results: The predictors included in the individualized prediction nomogram were chemical shift effect (CSE), nodal border, short-axis diameter of nodes, and minimum distance to rectal cancer or rectal wall. The nomogram showed good discrimination (C-index: 0.947; 95\% confidence interval: 0.920-0.974) and good calibration in the primary cohort. Decision curve analysis confirmed the clinical usefulness of the nomogram in predicting the status of each LN. For the prediction of LN status in the clinical validation cohort by readers 1 and 2, the areas under the curves using the nomogram were 0.890 and 0.841 , and the areas under the curves of readers using their experience were 0.754 and 0.704 , respectively. Diagnostic efficiency was significantly improved by using the nomogram $(\mathrm{P}<0.001)$.

Conclusions: The nomogram, which incorporates CSE, nodal location, short-axis diameter, and minimum distance to rectal cancer or rectal wall, can be conveniently applied in clinical practice to facilitate the prediction of LNM in patients with rectal cancer.

Keywords: Rectal cancer; magnetic resonance imaging (MRI); nomogram; lymph nodes (LNs); chemical shift effect (CSE)

Submitted Sep 08, 2020. Accepted for publication Feb 05, 2021.

doi: $10.21037 /$ qims-20-1049

View this article at: http://dx.doi.org/10.21037/qims-20-1049

\footnotetext{
^ ORCID: 0000-0002-2976-8414.
} 


\section{Introduction}

Colorectal cancer is one of the most common cancers worldwide, and in almost one-third of cases, the lesions occur in the rectum. The outcomes of patients with rectal cancer have improved significantly within the past 2 decades thanks to the development of multimodal therapeutic interventions (1). Lymph node (LN) staging is critical for therapeutic decision-making and predicting the prognosis of patients with rectal cancer. According to the National Comprehensive Cancer Network guidelines (2), it is recommended that patients with T1-2N0M0 rectal cancer undergo endoscopic mucosal resection or total mesorectal excision (TME). However, if the possibility of LN metastasis (LNM) exists, it is suggested that patients receive neoadjuvant chemoradiotherapy (nCRT), which is associated with a high incidence of adverse events and side-effects (e.g., radiation-induced injury, hematological toxicities, and long-term bowel dysfunction) (3-5).

Magnetic resonance imaging (MRI) has excellent soft tissue resolution and is widely regarded as the optimal non-invasive imaging tool for staging rectal cancer (6-9). Despite numerous efforts, the pre-treatment staging of LNs remains challenging (10-12). Size is of limited value in defining node-positive status, because $\mathrm{LN}$ enlargement can be caused by inflammation or reactive hyperplasia, while small LNs may contain metastatic tumor cells $(10,13)$. Morphological features, such as nodal borders or an internal signal (IS) pattern, are reported to be possible predictive factors of LNM $(14,15)$, but their predictive value remains controversial due to being observer dependent $(10,16)$.

F-18 fluorodeoxyglucose (FDG) positron emission tomography/computed tomography (PET/CT) has been found to be useful for the preoperative staging of rectal cancer by revealing metabolic information of the lesion $(17,18)$. However, F-18 FDG PET/CT also shows low sensitivity for the detection of LNM because of the partial volume effect, which spills out of the radioactive material into the background of lesions $<10 \mathrm{~mm}$ in size, leading to underestimation of the true standardized uptake value $(19,20)$.

Zhang et al. reported the chemical shift effect (CSE) along the border of LNs in MRI to be a potential predictor for distinguishing benign from metastatic LNs in a preliminary study with a small sample size (21). In that study, however, approximately one-third of small benign nodes had an absence of CSE, and these nodes were difficult to distinguish from metastatic nodes based on CSE. Therefore, this variable alone was not sufficient to differentiate metastatic LNs from benign $\mathrm{LNs}$ with confidence.

The aim of the present study was to investigate MRI variables that are potentially associated with metastasis, and to construct a risk-stratification nomogram with node-fornode histopathological validation of MR images. We also compared the predictive accuracy of the nomogram and subjective radiologist assessments, which are usually based on the predictors of nodal size, border, or IS, for nodal staging.

\section{Methods}

\section{Patients and study design}

The present prospective study was conducted on a primary cohort of patients (32 patients; 17 males and 15 females; mean age: 57.3 years; age range, $43-77$ years) between March 2015 and August 2016. The inclusion criteria were as follows: (I) diagnosis of rectal cancer by endoscopic biopsy and upcoming TME; (II) no history of anticancer therapy; and (III) no contraindications for MRI. The exclusion criteria were as follows: (I) patient received nCRT rather than direct surgery; or (II) patient underwent incision of the rectal specimen after TME.

An independent cohort of patients (182 patients; 122 males and 60 females; mean age: 58.1 years; age range, 21-84 years) who met the inclusion criteria was enrolled for clinical validation of the nomogram between September 2016 and June 2019. The inclusion criteria were as follows: (I) diagnosis of rectal cancer; (II) TME with no prior treatment before surgery; and (III) availability of preoperative MRI within 2 weeks prior to surgery.

The trial was conducted in accordance with the Declaration of Helsinki (as revised in 2013). The study was approved by the institutional ethics committee (No. NCC2015ST-27). Written informed consent was obtained from all primary cohort participants, and the requirement for consent was waived for participants in the validation cohort.

\section{MRI acquisition}

MRI was performed on a 3.0T system (Discovery MR 750; GE Healthcare, Waukesha, WI, USA) that utilized an 8 -element, phased-array, wrap-around surface coil. Patient preparation protocols together with MRI acquisition and 

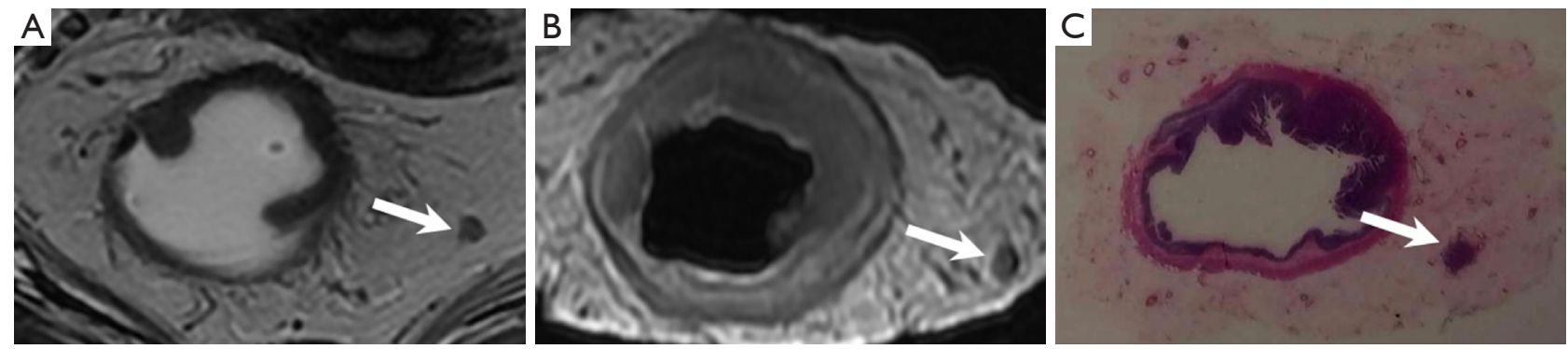

Figure 1 Metastatic lymph node harvested from a 62-year-old woman (white arrows). (A) Oblique axial $\mathrm{T}_{2}$-weighted in vivo magnetic resonance (MR) image; (B) $\mathrm{T}_{2}$-weighted ex vivo MR image; (C) whole-mount histological section.

protocols are described in Supplementary Appendix S1.

\section{Imaging analyses for the primary cobort}

MR images were reviewed by consensus by two radiologists to identify each mesorectal LN. The two radiologists independently evaluated the morphological factors, including nodal location, CSE, border, IS, and signal intensity (SI). Quantitative variables, including nodal size and minimum distance from the node to the rectal cancer or rectal wall (MinDR), were evaluated in consensus and measured precisely by the more experienced radiologist. Both radiologists were blinded to the clinical and histological results. All the variables are described in detail in Supplementary Appendix S2.

\section{Specimen MRI and histopathological evaluation for the primary cobort}

TME surgery and histological evaluation were carried out by a specialized surgeon and a dedicated pathologist with more than 20 years of experience in colorectal oncology and gastrointestinal pathology, respectively. Each resected specimen was initially fixed in formalin for 24 hours, and the circumferential resection plane was inked. The specimen was subsequently examined using MRI with the same preoperative protocols. After the ex vivo MRI examination, each specimen was transversely sliced perpendicular to the rectal lumen at intervals of $3 \mathrm{~mm}$. The sections were then stained with hematoxylin-eosin, and the status of each $\mathrm{LN}$ was categorized as benign or metastatic via light microscopic examination.

Slices were compared with in vivo and ex vivo MR images to obtain a precise slice-for-section match. The slice and location of each LN were matched with the corresponding MR image (when visible) to enable a nodefor-node comparison (Figure 1). On the basis of Lambregts et al.'s study, special attention was paid to the size and position of the nodes in relation to the tumor, rectal wall, mesorectal fascia, small blood vessels, and adjacent LNs (22). Although the specimen MRI was used for nodal validation of in vivo MRI and histopathology, it was not used for the characteristic evaluation of the nodes.

The LNs on preoperative MRI, which were exactly matched with histopathological results, were classified as benign or metastatic. The MR parameters were analyzed statistically and incorporated to build the predictive nomogram.

\section{Evaluation of LN staging for the clinical validation cohort}

LN staging for the clinical validation cohort was evaluated independently by two experienced radiologists (readers 1 and 2). First, the two readers evaluated the LN staging of each patient according to their daily experience, generally on the basis of the predictors of size, border, and IS of the LNs, as recommended by the European Society of Gastrointestinal and Abdominal Radiology (23). Two weeks later, the 182 cases were reordered, and the readers were trained to cognize the nomogram and reassess LN staging based on the nomogram. The detailed approach was as follows. First, the cut-off value for the differentiation of benign and metastatic nodes was obtained from the nomogram that was developed using the prospective cohort. Second, to acquire the total points of each LN, the readers evaluated the LNs of each patient according to the nomogram. LNs with total points lower or higher than the cut-off value were categorized as benign or metastatic nodes, respectively. Finally, the LN stage of each patient was decided based on the number of evaluated metastatic 
nodes (e.g., N0, N1, or N2) according to the 8th edition of the American Joint Committee on Cancer staging system.

\section{Statistical analysis}

To investigate the risk factors for LNM in the primary cohort, the significance of each variable was assessed using a univariable analysis. According to the results of a test for normal distribution, the associations between the continuous variables and LNM were assessed using the $t$-test or the Mann-Whitney U-test. Categorical and ordinal variables were assessed using the $\chi^{2}$ test or Fisher's exact test. $\mathrm{P}<0.05$ was considered to be statistically significant. Interobserver agreement between the two radiologists who evaluated the primary cohort was quantified by kappa statistics as follows: $<0.20$, slight; $0.21-0.40$, fair; $0.41-0.60$, moderate; $0.61-0.80$, substantial; and $>0.80$, almost perfect reproducibility.

All variables found to be associated with $\mathrm{LNM}$ at $\mathrm{P}<0.1$ in the univariable analysis were included in the multivariable logistic analysis. Backward stepwise selection was applied with Akaike's information criterion as the stopping rule. A nomogram was formulated based on the results of the multivariable logistic regression analysis to predict the status of each LN in the primary cohort.

The calibration curve, which was obtained by plotting the actual LNM probability against the nomogram-predicted LNM probability, was used to assess the calibration of the nomogram in the primary cohort. The Hosmer-Lemeshow test was used to assess the goodness of fit of the calibration curve (a significant test statistic implies that the nomogram does not perfectly calibrate) (24). Harrell's C-index was measured to quantify the discriminatory performance of the nomogram, and the nomogram was subjected to bootstrapping validation (1,000 bootstrapping resamples) to achieve a relatively corrected $\mathrm{C}$-index.

To assess the clinical usefulness of the nomogram for predicting the status of each $\mathrm{LN}$ in the primary cohort, a decision curve analysis (DCA) was conducted by calculating the net benefits at different threshold probabilities (25).

To verify the clinical usefulness of the nomogram for predicting LN staging on a per-patient basis, the results of LN staging based on the radiologists' experience and the nomogram were compared with histopathological findings in the clinical validation cohort. The optimal cut-off value for the differentiation of benign and metastatic nodes was determined based on the Youden index. The sensitivity, specificity, and accuracy of the nomogram for LN staging were calculated, and the area under the curve (AUC) was used to assess its diagnostic performance. The Delong method was applied to compare the AUCs. Statistical analyses were conducted with R version 3.6.1 (http://www. Rproject.org).

\section{Results}

\section{Study cohorts}

A total of 51 consecutive patients with rectal cancer underwent preoperative MRI. Nineteen patients were excluded from the study because they received nCRT $(n=13)$ or the surgeons performed a longitudinal incision of the specimen after surgery $(\mathrm{n}=6)$. Finally, 32 patients $(17$ males and 15 females; mean age: 57.3 years; age range, $43-77$ years) were recruited and included in the primary cohort. The interval between preoperative MRI and TME was less than 2 weeks (mean days: 5 days; range, $2-12$ days).

A total of 523 nodes from the 32 patients $(22 \pm 3$ nodes per patient; range, 7-40) were harvested. Forty-nine nodes in ten patients were metastatic, and 474 nodes were benign. Among the 523 nodes in total, 324 were matched exactly with the nodes on MR images, and 199 nodes could not be matched. These 199 nodes, including 7 metastatic nodes, were excluded from the following analyses. The remaining 324 nodes ( 42 metastatic and 282 benign) were collected for the node-by-node evaluation.

\section{Univariable analysis}

Table 1 summarizes the results of the univariable analysis that was used to identify the MR parameters for predicting LNM. Nodal location, CSE, border, IS, SI, short-axis diameter (SD), and MinDR were significantly different between benign and metastatic LNs $(\mathrm{P}<0.05)$; thus, these factors were included in the multivariable logistic regression analysis.

A total of $95.2 \%(40 / 42)$ of the metastatic LNs and $62.4 \%(176 / 282)$ of the benign LNs were located ipsilateral to the primary tumor $(\mathrm{P}<0.001)$. A total of $97.6 \%(41 / 42)$ of the metastatic LNs showed an irregular or absent CSE, while $72.3 \%$ (204/282) of the benign LNs showed a regular CSE along the border $(\mathrm{P}<0.001)$ (Figure 2).

\section{Interobserver agreement}

The kappa values for interobserver agreement were 0.931 (nodal location), 0.787 (CSE), 0.629 (border), 0.577 (IS), 
Table 1 Comparison of variables for differentiating between metastatic and benign lymph nodes (LNs) using univariable analysis

\begin{tabular}{|c|c|c|c|}
\hline Variables & Metastatic LNs $(n=42)$ & Benign LNs $(n=282)$ & $P$ value \\
\hline Ipsilateral and at tumor height & $24(57.1)$ & $77(27.3)$ & \\
\hline Ipsilateral and outside tumor height & $16(38.1)$ & $99(35.1)$ & \\
\hline Contralateral and at tumor height & $1(2.4)$ & $29(10.3)$ & \\
\hline CSE, n (\%) & & & $<0.001^{\ddagger}$ \\
\hline Regular & $1(2.4)$ & $204(72.3)$ & \\
\hline Irregular & $8(19.0)$ & $33(11.7)$ & \\
\hline Absent & $33(78.6)$ & $45(16.0)$ & \\
\hline Poorly defined & $28(66.7)$ & $71(25.2)$ & \\
\hline Internal signal pattern (n) & & & $0.023^{\ddagger}$ \\
\hline Homogenous & $18(42.9)$ & $173(61.3)$ & \\
\hline Heterogenous & $24(57.1)$ & $109(38.7)$ & \\
\hline Signal intensity, n (\%) & & & $<0.001^{\dagger}$ \\
\hline Hyperintensity & $14(33.3)$ & 201 (71.3) & \\
\hline Isointensity & $18(42.9)$ & $55(19.5)$ & \\
\hline Hypointensity & $10(23.8)$ & $26(9.2)$ & \\
\hline
\end{tabular}

${ }^{\dagger}$, Fisher's exact test; ${ }^{\ddagger}, \chi^{2}$ test; ${ }^{\S}$, Mann-Whitney U-test. unless otherwise indicated, data are presented as numbers of LNs (percentages). CSE, chemical shift effect; IQR, interquartile range; LD, long-axis diameter; MinDR, minimum distance to rectal cancer or rectal wall; SD, short-axis diameter; S/L, short- to long-axis diameter ratio.

and $0.581(\mathrm{SI})$.

\section{Multivariable analysis and nomogram development}

Results from the multivariable logistic regression analysis revealed that nodal location, CSE, SD, and MinDR were independent risk factors for LNM (Table 2). A model incorporating the above independent factors was developed and presented as a nomogram, and a web-based calculator was also developed (Figure 3). The web-based calculator is available at https://rectalcancernom.shinyapps.io/ DynNomapp/.
The calibration curve of the nomogram evidenced good agreement between the LNM rates that were predicted by the nomogram and the actual probabilities of LNM in the primary cohort (Figure 4). The Hosmer-Lemeshow test yielded a non-significant statistical result $(\mathrm{P}=0.911)$, suggesting that there was no departure from the perfect fit. The calibration curve and a non-significant HosmerLemeshow test result indicated good calibration of the nomogram. The C-index for the nomogram was 0.947 [95\% confidence interval (CI): 0.920-0.974] in the primary cohort and was confirmed to be 0.931 via 1,000 bootstrapping validation, revealing good discrimination of the nomogram. 


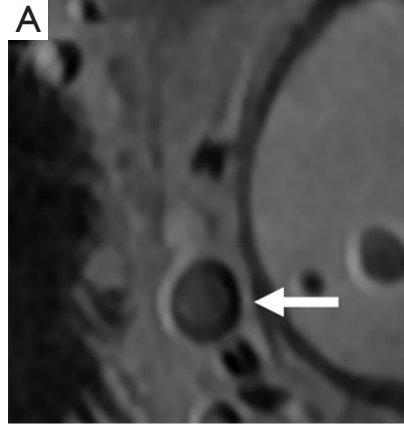

CSE: regular
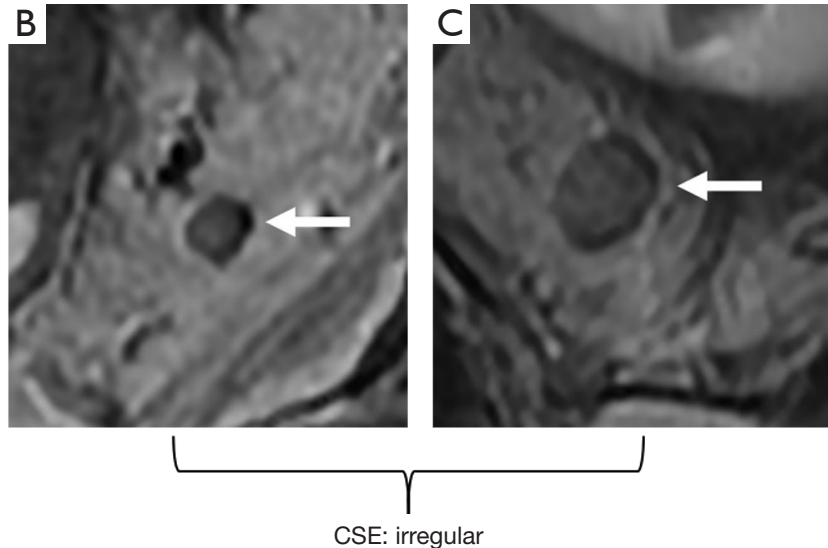

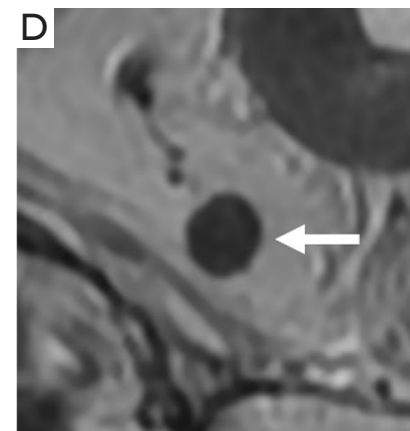

CSE: absent

Figure 2 Three subtypes of chemical shift effect (CSE) on $\mathrm{T}_{2}$-weighted images (white arrows). (A) Regular CSE; CSE at the border of lymph node is complete and smooth. (B,C) Irregular CSE; CSE at the border of the lymph node is irregular, incomplete, or interrupted. (D) Absent CSE; CSE at the border of the lymph node is absent.

Table 2 Risk factors for lymph node metastasis in rectal cancer

\begin{tabular}{|c|c|c|c|}
\hline Variable & $\beta$ & Odds ratio (95\% Cl) & $P$ value \\
\hline \multicolumn{4}{|l|}{ Location } \\
\hline Ipsilateral and at tumor height & - & 1.000 & - \\
\hline Ipsilateral and outside tumor height & -0.500 & $0.606(0.222,1.604)$ & 0.318 \\
\hline Contralateral and at tumor height & -2.435 & $0.088(0.004,0.581)$ & 0.033 \\
\hline \multicolumn{4}{|l|}{ CSE } \\
\hline Regular & - & 1.000 & - \\
\hline Irregular & 4.195 & $66.381(10.076,1,357.976)$ & $<0.001$ \\
\hline Absent & 5.859 & $350.435(55.849,7,441.252)$ & $<0.001$ \\
\hline
\end{tabular}

$\mathrm{Cl}$, confidence interval; CSE, chemical shift effect; MinDR, minimum distance to rectal cancer or rectal wall; SD, short-axis diameter.

The result of the DCA for the nomogram is presented in Figure 5. The DCA showed that the proposed nomogram for predicting LNM was advantageous over the treat-allnodes scheme or the treat-none scheme when the threshold probability ranged from $0 \%$ to $100 \%$. This finding indicated that the nomogram had a good performance with respect to clinical application.

\section{Validation of the nomogram}

The results of LN staging for the 182 clinical validation cohort patients are listed in Table 3. The accuracy of readers 1 and 2 for predicting LN staging based on their experience was $67.6 \%$ and $57.7 \%$, respectively, compared with an accuracy of $80.2 \%$ and $76.4 \%$, respectively, when using the nomogram. On this basis, the LN status of each patient was classified as positive or negative. The predictive efficacy of each reader is shown in Table 4. For readers 1 and 2, the AUCs for predicting LN status based on experience were 0.754 (95\% CI: 0.692-0.816) and 0.704 (95\% CI: 0.637-0.770), respectively, and the AUCs when using the nomogram were 0.890 (95\% CI: 0.844-0.936) and 


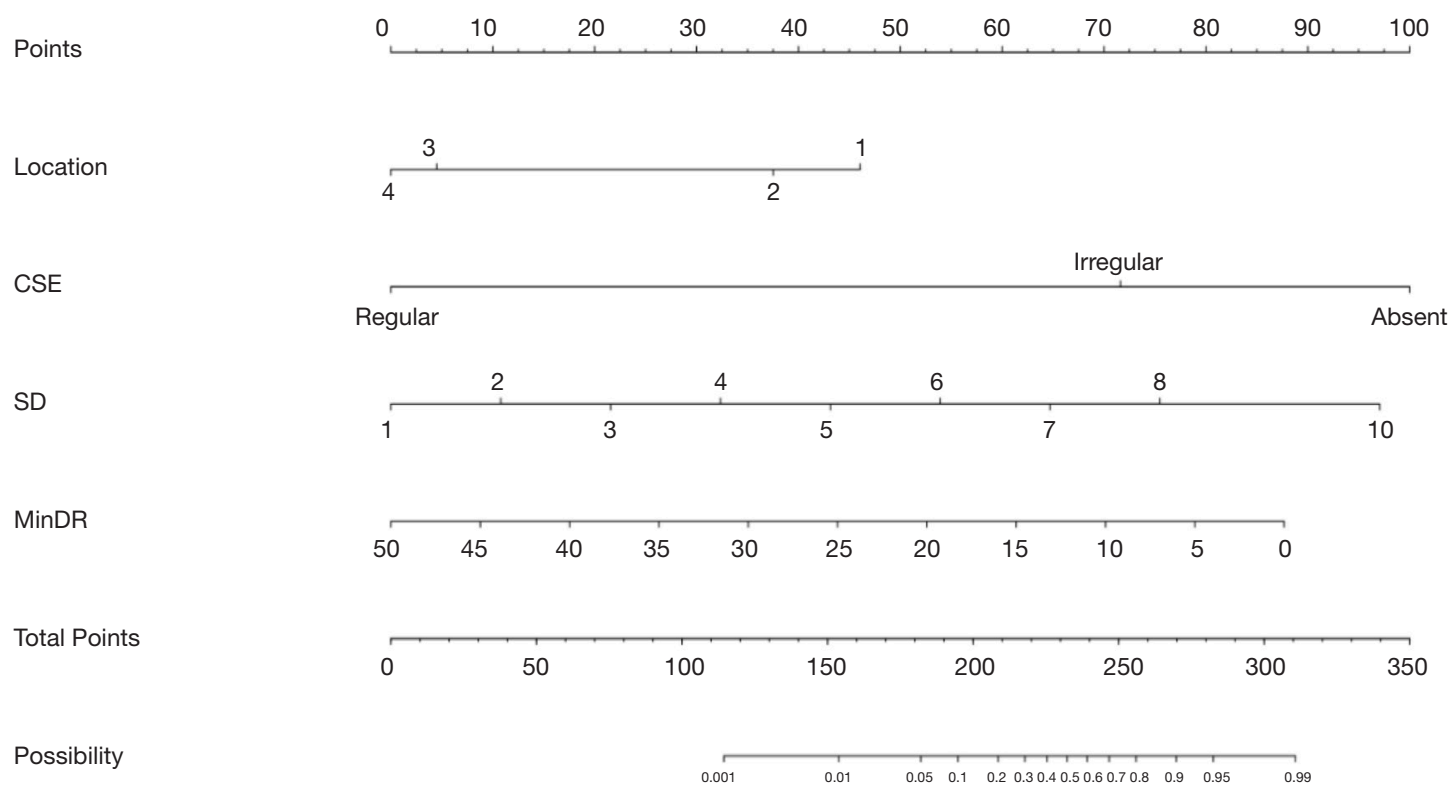

\section{A magnetic resonance imaging (MRI)-based nomogram for predicting lymph node metastasis in rectal cancer: a node-for-node comparative study of MRI and histopathology}
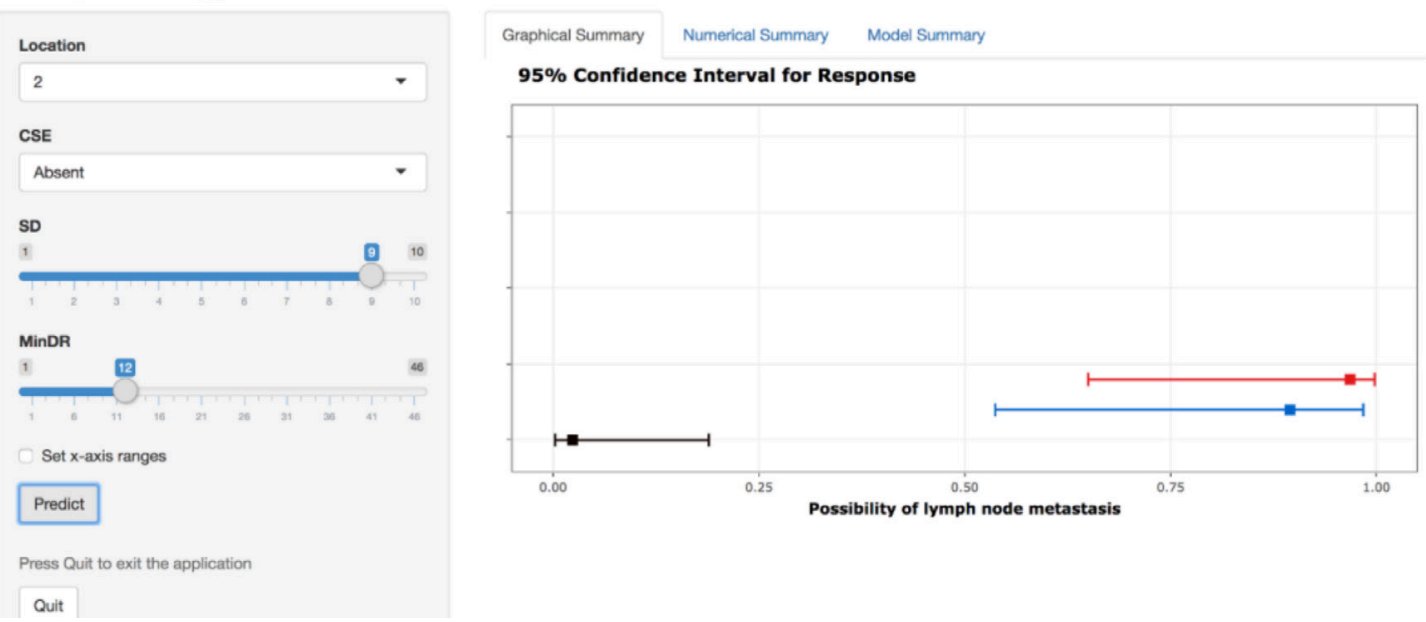

Figure 3 Nomogram and corresponding online calculator for predicting lymph node (LN) status. The nomogram incorporates nodal location, chemical shift effect, short-axis dimension, and minimal distance to rectal cancer or rectal wall. To use the nomogram, an individual $\mathrm{LN}$ value is located on each variable axis, and a line is drawn upward to determine the number of points received for each variable value. The sum of these numbers is located on the total points axis, and a line is drawn downward to the LN status axis to determine the likelihood of LN status. The online tool is available at https://rectalcancernom.shinyapps.io/DynNomapp/. Location 1, ipsilateral and at tumor height; location 2, ipsilateral and outside tumor height; location 3, contralateral and at tumor height; and location 4, contralateral and outside tumor height. 


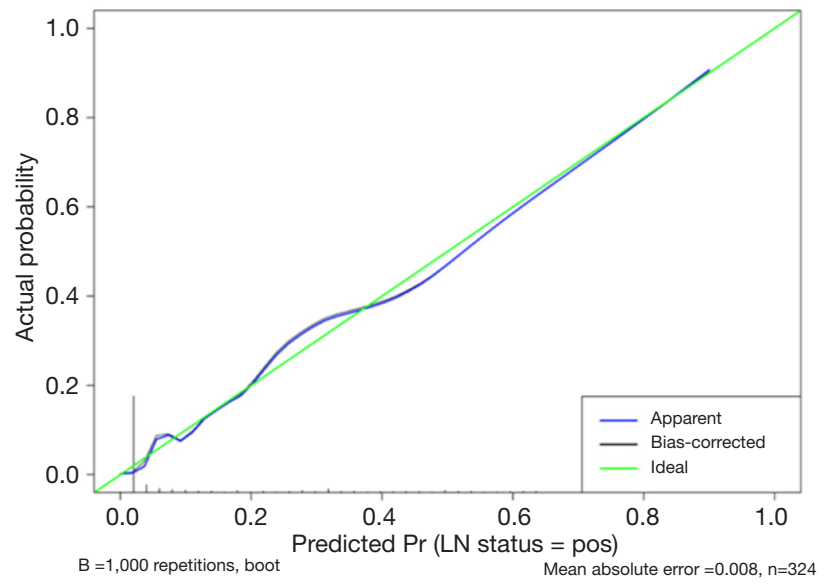

Figure 4 Calibration curve of the nomogram. Calibration curve depicts the calibration of the nomogram in terms of the agreement between the predicted lymph node (LN) metastasis risk and the actual pathological LN outcomes. Y-axis represents the actual LN metastasis (LNM) rate, and the $\mathrm{x}$-axis represents the predicted LNM risk. Green line represents a perfect prediction by an ideal model. Blue line represents the predictive performance of the nomogram, and a closer fit to the green line represents a better prediction.

0.841 (95\% CI: 0.787-0.894), respectively. Therefore, the AUCs based on the nomogram were significantly greater than those based on reader experience $(\mathrm{P}<0.001)$ for both readers.

\section{Discussion}

MRI is an essential diagnostic tool for rectal cancer staging; however, currently, it has only moderate accuracy for predicting LNM in patients with rectal cancer (5,26-28). This is a significant shortcoming considering the implications of inaccurate treatment selection and prognosis (13). Current debates surrounding LNM focus on the following aspects $(7,14,29)$ : (I) the optimal nodal size cut-off for predicting LN status; (II) whether the border or the IS of nodes represents an independent risk factor for metastasis; and (III) whether risk factors other than size are important parameters.

We developed an easy-to-use nomogram for the preoperative individualized prediction of LNM in patients with rectal cancer and validated the nomogram in a clinical validation cohort. The nomogram incorporated CSE (with the highest weighting), nodal location, SD, and

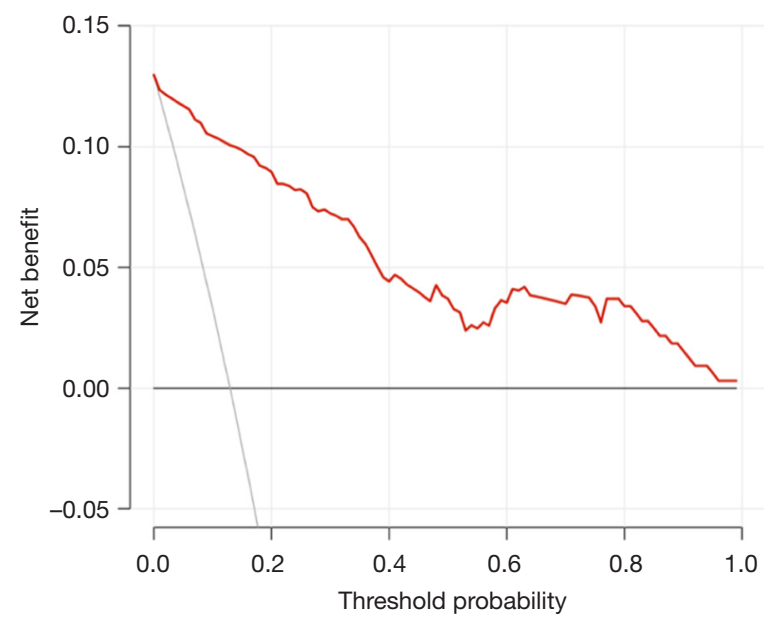

Figure 5 Decision curve analysis (DCA) for the nomogram. Y-axis measures the net benefit, and the $\mathrm{x}$-axis represents the threshold probability. Red line represents the predictive nomogram. Gray line represents the assumption that all lymph nodes (LNs) are metastatic. Black line represents the assumption that no LNs are metastatic. Threshold probability refers to the point at which the expected benefit of treatment is equivalent to the expected benefit of avoiding treatment. For instance, if the possibility of metastasis of an $\mathrm{LN}$ is over the threshold probability, then a treatment strategy for LN metastasis (LNM) should be adopted. DCA in the primary cohort showed that if the threshold probability is between 0 and 1.0, using the nomogram to predict LNM is more beneficial than either the treat-all-nodes scheme or the treat-none scheme.

MinDR. The predictive accuracy of LN staging using the nomogram was statistically superior to that based on only the experiences of the radiologists.

In the present study, more than two-thirds of benign LNs showed a regular CSE at the edge; however, only 1 of the 42 metastatic LNs exhibited a regular CSE at the edge. Therefore, we concluded that CSE was an important factor for differentiating between benign and metastatic nodes.

CSE is known to be caused by Larmor frequency shifts in water and fat protons. It emerges as a black border at 1 fat-water interface and a bright border at the opposite interface. For benign LNs, the fat-water interface is formed by the lymph fluid in the subcapsular sinus and the fat in the mesorectum. However, in metastatic LNs, infiltration of tumor cells initially occurs via the afferent lymphatic vessels into the subcapsular sinus, and the junction between the afferent lymphatic vessels and the subcapsular sinus is the most common place for the gathering of tumor foci. 
Table 3 Results of lymph node staging by readers

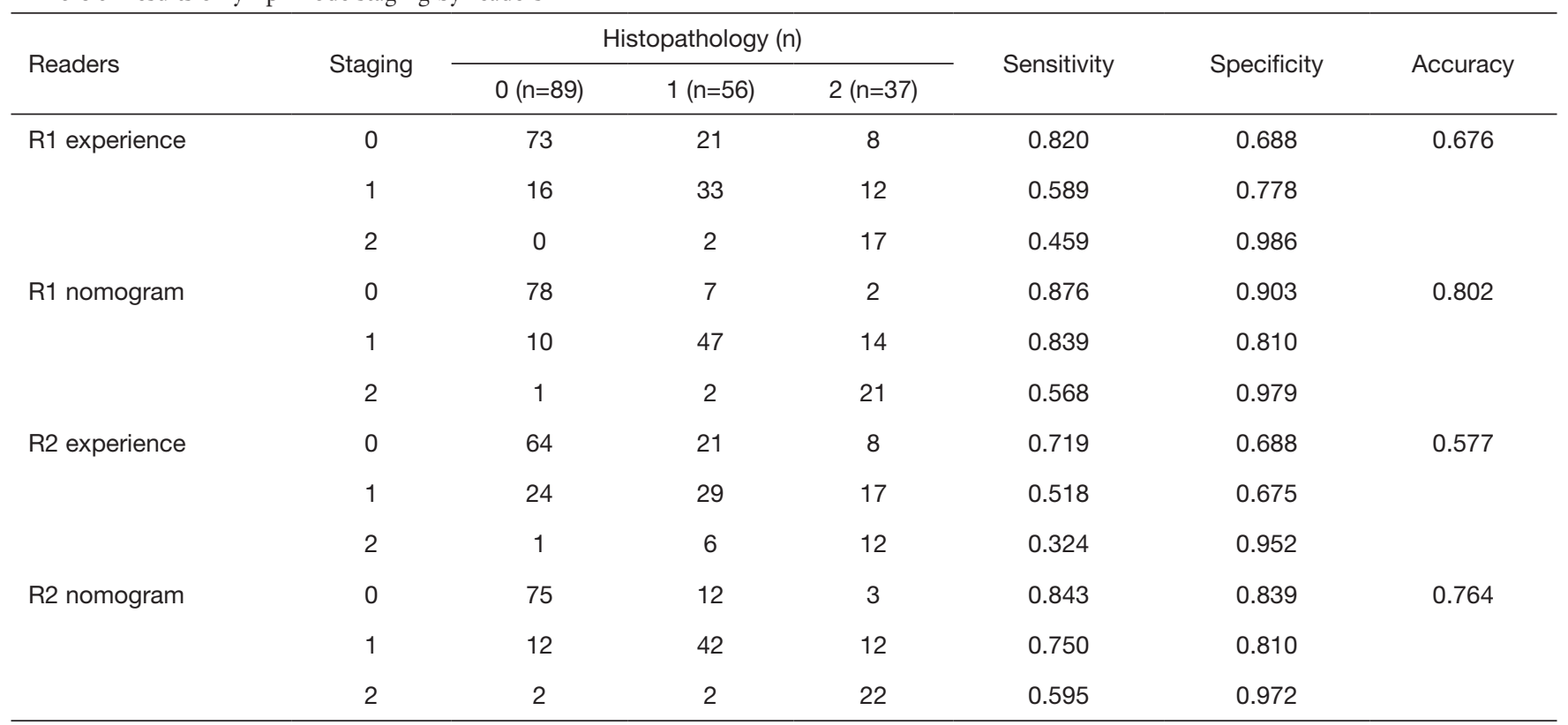

$\mathrm{R} 1$, reader 1; R2, reader 2 .

Table 4 Predictive efficacy for discriminating positive and negative lymph nodes

\begin{tabular}{lccccc}
\hline Readers & AUC & Sensitivity & Specificity & PPV & NPV \\
\hline R1 experience & 0.754 & 0.688 & 0.820 & 0.800 & 0.716 \\
R1 nomogram & 0.890 & 0.903 & 0.876 & 0.884 & 0.897 \\
R2 experience & 0.704 & 0.688 & 0.719 & 0.719 & 0.688 \\
R2 nomogram & 0.841 & 0.839 & 0.843 & 0.848 & 0.833 \\
\hline
\end{tabular}

AUC, area under the curve; NPV, negative predictive value; PPV, positive predictive value; R1, reader 1; R2, reader 2.

Therefore, the original fat-water interface is destroyed by tumor invasion (30-32). In the present study, CSE showed excellent sensitivity for defining metastatic LNs. However, it should be noted that $27.7 \%$ of benign LNs showed an irregular or absent CSE, and therefore, may mimic metastatic LNs. The restriction of spatial resolution likely affects the observation of CSE in some nodes, especially small ones (21).

In the present study, the majority of metastatic nodes were located ipsilateral to the primary tumor. Furthermore, nodes that were close to the primary tumor or the rectal wall were more likely to be involved. This result is consistent with reports on the nodal drainage pathway $(33,34)$.

Nodal size was incorporated into the nomogram with a relatively low weighting. A node with a larger SD was more likely to be metastatic. Nodal size is more effective as a weighting factor than the nodal size cut-off value for determining LN status. However, previous studies have reported that an $\mathrm{SD} \geq 9 \mathrm{~mm}$ could be considered as a relatively reliable morphological criterion for predicting LNM $(13,23)$. In our study, two LNs with $\mathrm{SD} \geq 9 \mathrm{~mm}$ were detected in the primary cohort, and both were found to be metastatic.

The findings of our univariable analysis indicated that the nodal border, IS, and SI were significant factors for predicting LN status. However, these variables lost their predictive potential in the multivariable logistic regression analysis. In the present study, it was observed that a regular CSE usually appeared at the well-defined border of the LNs. This may, in part, be the reason why the border was excluded in the multivariable analysis. Prediction of the 
IS and SI of LNs was partly influenced by the subjective judgment of the readers with only moderate interobserver reproducibility (kappa value $=0.577$ and 0.581 , respectively), which proved that IS and SI were not optimal predictors for clinical practice.

Considering that the purpose of constructing the nomogram was to improve the diagnostic accuracy of LN staging for patients with rectal cancer in clinical practice, clinical validation was performed in the present study, to verify the performance of the nomogram for predicting the LN staging of each patient instead of predicting the status of each LN. Moreover, in the clinical setting, radiologists usually predict LN staging on the basis of the size, border, or IS of the LNs. For clinical validation of the nomogram, we compared its predictive efficacy with the predictive efficacy of using the radiologists' experience. The results suggested that the nomogram, which is more objective and precise, can significantly improve diagnostic accuracy in daily clinical practice.

Among cases of incorrectly predicted LN staging in the present study, a lot of cases were under-staged, either based on the nomogram or radiologist's experience. Some small LNs were easily missed on MRI, despite thin slice thickness and high spatial resolution being applied. This phenomenon appeared particularly in low rectal cancer, located in a relatively narrow space with minimal mesorectal fat $(7,35)$. For such cases, other information, such as primary tumor staging or mesorectal fascia status, may facilitate LN staging.

There were some limitations to our study. First, the nomogram was established based on data obtained from a single institution. Second, some patients who were diagnosed with locally advanced rectal cancer on preoperative MRI underwent direct surgery because of under-staged or refused to accept nCRT in our study. Third, due to the limitation of the 3 -mm slice thickness for T2-weighted imaging, quite a few nodes $<3 \mathrm{~mm}$ could not be detected by MRI, including 7 of 199 (3.5\%) nodes with metastasis. Furthermore, water loss at the edge of the resected specimen led to the reduction of the mesorectal signal, and some LNs showed isointensity on T2-weighted imaging compared with mesorectal fat. Although diffusionweighted imaging was also performed for the resected specimen in the present study, it was still difficult to identify these nodes. Assessment of LN status using our nomogram was done based on visual evaluation; therefore, nodes with metastasis that could not be detected by MRI may be missed. Quantitative image analysis techniques, such as machine learning, may have the potential to further improve the predictive ability of LN status. Fourth, the number of metastatic LNs in the primary cohort was relatively low compared with the number of benign LNs, as it is usually recommended that patients with distinct LNM on pretreatment MRI receive nCRT rather than surgery. Fifth, tumor deposits were not assessed in the study. Sixth, the best validation for the present study was performed using both node-by-node analysis and per-patient analysis. In the clinical validation cohort, histological whole-mount specimens were not assessed, and the status of each specific LN could not be defined. Therefore, a node-by-node validation was not performed in the present study. Despite these limitations, when the nomogram was used to predict preoperative $\mathrm{LN}$ staging, we demonstrated that it increased the diagnostic efficiency compared to subjective assessments performed by skilled radiologists. Objective evaluation tools, such as our nomogram, are needed in the clinical settings to achieve exact $\mathrm{LN}$ staging to guide individualized treatment. In the future, multicenter studies with a larger sample size should be conducted to verify the diagnostic performance of the nomogram.

In conclusion, in the present study, we have proposed a nomogram that incorporates CSE, a new risk factor, and assigns it the highest weighting. The nomogram also includes the predictors of nodal location, SD, and MinDR of LNs. Overall, our findings demonstrate that this nomogram can be conveniently used in the clinical setting to facilitate the prediction of LNM in patients with rectal cancer.

\section{Acknowledgments}

Funding: This research is supported by the National Natural Science Foundation of China (grant number 81971589); CAMS Innovation Fund for Medical Sciences (grant number 2018-I2M-AI-008).

\section{Footnote}

Conflicts of Interest: All authors have completed the ICMJE uniform disclosure form (available at http://dx.doi. org/10.21037/qims-20-1049). All authors report grants from The National Natural Science Foundation of China, grants from CAMS Innovation Fund for Medical Sciences, during the conduct of the study.

Ethical Statement: The trial was conducted in accordance 
with the Declaration of Helsinki (as revised in 2013). The study was approved by the institutional ethics committee (No. NCC2015ST-27). Written informed consent was obtained from all primary cohort patients and waived for the validation cohort patients.

Open Access Statement: This is an Open Access article distributed in accordance with the Creative Commons Attribution-NonCommercial-NoDerivs 4.0 International License (CC BY-NC-ND 4.0), which permits the noncommercial replication and distribution of the article with the strict proviso that no changes or edits are made and the original work is properly cited (including links to both the formal publication through the relevant DOI and the license). See: https://creativecommons.org/licenses/by-nc-nd/4.0/.

\section{References}

1. Wiegering A, Isbert C, Dietz UA, Kunzmann V, Ackermann S, Kerscher A, Maeder U, Flentje M, Schlegel N, Reibetanz J, Germer CT, Klein I. Multimodal therapy in treatment of rectal cancer is associated with improved survival and reduced local recurrence - a retrospective analysis over two decades. BMC Cancer 2014;14:816.

2. National Comprehensive Cancer Network. NCCN clinical practice guidelines in Oncology: Rectal Cancer (2020.V1). [2021-01-18]. Available online: https://www. nccn.org/professionals/physician_gls/pdf/rectal.pdf

3. Lai LL, Fuller CD, Kachnic LA, Thomas CR Jr. Can pelvic radiotherapy be omitted in select patients with rectal cancer? Semin Oncol 2006;33:S70-4.

4. Rahbari NN, Elbers H, Askoxylakis V, Motschall E, Bork U, Buchler MW, Weitz J, Koch M. Neoadjuvant radiotherapy for rectal cancer: meta-analysis of randomized controlled trials. Ann Surg Oncol 2013;20:4169-82.

5. Rödel C, Martus P, Papadoupolos T, Fuzesi L, Klimpfinger M, Fietkau R, Liersch T, Hohenberger W, Raab R, Sauer R, Wittekind C. Prognostic significance of tumor regression after preoperative chemoradiotherapy for rectal cancer. J Clin Oncol 2005;23:8688-96.

6. Nougaret S, Reinhold C, Mikhael HW, Rouanet P, Bibeau F, Brown G. The use of MR imaging in treatment planning for patients with rectal carcinoma: have you checked the "DISTANCE"? Radiology 2013;268:330-44.

7. Horvat N, Carlos Tavares Rocha C, Clemente Oliveira B, Petkovska I, Gollub MJ. MRI of rectal cancer: tumor staging, imaging techniques, and management. Radiographics 2019;39:367-87.
8. Peng $\mathrm{Y}$, Tang H, Meng X, Shen Y, Hu D, Kamel I, Li Z. Histological grades of rectal cancer: whole-volume histogram analysis of apparent diffusion coefficient based on reduced field-of-view diffusion-weighted imaging. Quant Imaging Med Surg 2020;10:243-56.

9. Tang X, Luo Y, Zhang S, Xia L, Gong J. Local staging of rectal cancer using fused high resolution diffusion weighted imaging and modified MR rectography. Quant Imaging Med Surg 2019;9:1592-6.

10. Gröne J, Loch FN, Taupitz M, Schmidt C, Kreis ME. Accuracy of various lymph node staging criteria in rectal cancer with magnetic resonance imaging. J Gastrointest Surg 2018;22:146-53.

11. Doyon F, Attenberger UI, Dinter DJ, Schoenberg SO, Post S, Kienle P. Clinical relevance of morphologic MRI criteria for the assessment of lymph nodes in patients with rectal cancer. Int J Colorectal Dis 2015;30:1541-6.

12. Chen XL, Chen GW, Pu H, Yin LL, Li ZL, Song B, Li H. DWI and T2-weighted MRI volumetry in resectable rectal cancer: correlation with lymphovascular invasion and lymph node metastases. AJR Am J Roentgenol 2019. [Epub ahead of print]. doi: 10.2214/AJR.18.20564.

13. Nougaret S, Jhaveri K, Kassam Z, Lall C, Kim DH. Rectal cancer MR staging: pearls and pitfalls at baseline examination. Abdom Radiol (NY) 2019;44:3536-48.

14. Brown G, Richards CJ, Bourne MW, Newcombe RG, Radcliffe AG, Dallimore NS, Williams GT. Morphologic predictors of lymph node status in rectal cancer with use of high-spatial-resolution MR imaging with histopathologic comparison. Radiology 2003;227:371-7.

15. Kim JH, Beets GL, Kim MJ, Kessels AG, Beets-Tan RG. High-resolution MR imaging for nodal staging in rectal cancer: are there any criteria in addition to the size? Eur J Radiol 2004;52:78-83.

16. Akasu T, Iinuma G, Takawa M, Yamamoto S, Muramatsu Y, Moriyama N. Accuracy of high-resolution magnetic resonance imaging in preoperative staging of rectal cancer. Ann Surg Oncol 2009;16:2787-94.

17. Kijima S, Sasaki T, Nagata K, Utano K, Lefor AT, Sugimoto H. Preoperative evaluation of colorectal cancer using CT colonography, MRI, and PET/CT. World J Gastroenterol 2014;20:16964-75.

18. Caglar M, Yener C, Karabulut E. Value of CT, FDG PET-CT and serum tumor markers in staging recurrent colorectal cancer. Int J Comput Assist Radiol Surg 2015; 10:993-1002.

19. Bae SU, Won KS, Song BI, Jeong WK, Baek SK, Kim HW. Accuracy of F-18 FDG PET/CT with optimal cut- 
offs of maximum standardized uptake value according to size for diagnosis of regional lymph node metastasis in patients with rectal cancer. Cancer Imaging 2018;18:32.

20. Kim DJ, Kim JH, Ryu YH, Jeon TJ, Yu JS, Chung JJ. Nodal staging of rectal cancer: high-resolution pelvic MRI versus 18F-FDGPET/CT. J Comput Assist Tomogr 2011;35:531-4.

21. Zhang H, Zhang C, Zheng Z, Ye F, Liu Y, Zou S, Zhou C. Chemical shift effect predicting lymph node status in rectal cancer using high-resolution MR imaging with node-fornode matched histopathological validation. Eur Radiol 2017;27:3845-55.

22. Lambregts DM, Heijnen LA, Maas M, Rutten IJ, Martens MH, Backes WH, Riedl RG, Bakers FC, Cappendijk VC, Beets GL, Beets-Tan RG. Gadofosveset-enhanced MRI for the assessment of rectal cancer lymph nodes: predictive criteria. Abdom Imaging 2013;38:720-7.

23. Beets-Tan RGH, Lambregts DMJ, Maas M, Bipat S, Barbaro B, Curvo-Semedo L, Fenlon HM, Gollub MJ, Gourtsoyianni S, Halligan S, Hoeffel C, Kim SH, Laghi A, Maier A, Rafaelsen SR, Stoker J, Taylor SA, Torkzad MR, Blomqvist L. Magnetic resonance imaging for clinical management of rectal cancer: Updated recommendations from the 2016 European Society of Gastrointestinal and Abdominal Radiology (ESGAR) consensus meeting. Eur Radiol 2018;28:1465-75.

24. Kramer AA, Zimmerman JE. Assessing the calibration of mortality benchmarks in critical care: The HosmerLemeshow test revisited. Crit Care Med 2007;35:2052-6.

25. Cui Y, Yang X, Shi Z, Yang Z, Du X, Zhao Z, Cheng X. Radiomics analysis of multiparametric MRI for prediction of pathological complete response to neoadjuvant chemoradiotherapy in locally advanced rectal cancer. Eur Radiol 2019;29:1211-20.

26. Halefoglu AM, Atasoy ST, Sakiz D, Baykan A. Accuracy of thin-section magnetic resonance imaging with a pelvic phased-array coil in the local staging of rectal cancer. J Comput Assist Tomogr 2013;37:58-64.

27. Al-Sukhni E, Milot L, Fruitman M, Beyene J, Victor JC, Schmocker S, Brown G, McLeod R, Kennedy E.

Cite this article as: Liu Y, Wan L, Peng W, Zou S, Zheng Z, Ye F, Jiang J, Ouyang H, Zhao X, Zhang H. A magnetic resonance imaging (MRI)-based nomogram for predicting lymph node metastasis in rectal cancer: a node-for-node comparative study of MRI and histopathology. Quant Imaging Med Surg 2021;11(6):2586-2597. doi: 10.21037/qims-20-1049
Diagnostic accuracy of MRI for assessment of T category, lymph node metastases, and circumferential resection margin involvement in patients with rectal cancer: a systematic review and meta-analysis. Ann Surg Oncol 2012;19:2212-23.

28. Gao Y, Li J, Ma X, Wang J, Wang B, Tian J, Chen G. The value of four imaging modalities in diagnosing lymph node involvement in rectal cancer: an overview and adjusted indirect comparison. Clin Exp Med 2019;19:225-34.

29. Kotanagi H, Fukuoka T, Shibata Y, Yoshioka T, Aizawa O, Saito Y, Tur GE, Koyama K. The size of regional lymph nodes does not correlate with the presence or absence of metastasis in lymph nodes in rectal cancer. J Surg Oncol 1993;54:252-4.

30. Diaz LK, Hunt K, Ames F, Meric F, Kuerer H, Babiera G, Ross M, Singletary E, Middleton LP, Symmans WF, Krishnamurthy S, Sahin A, Sneige N, Gilcrease MZ. Histologic localization of sentinel lymph node metastases in breast cancer. Am J Surg Pathol 2003;27:385-9.

31. Hayashi K, Jiang P, Yamauchi K, Yamamoto N, Tsuchiya H, Tomita K, Moossa AR, Bouvet M, Hoffman RM. Realtime imaging of tumor-cell shedding and trafficking in lymphatic channels. Cancer Res 2007;67:8223-8.

32. Servais EL, Colovos C, Bograd AJ, White J, Sadelain M, Adusumilli PS. Animal models and molecular imaging tools to investigate lymph node metastases. J Mol Med (Berl) 2011;89:753-69.

33. Kaur H, Ernst RD, Rauch GM, Harisinghani M. Nodal drainage pathways in primary rectal cancer: anatomy of regional and distant nodal spread. Abdom Radiol (NY) 2019;44:3527-35.

34. Steup WH, Moriya Y, van de Velde CJ. Patterns of lymphatic spread in rectal cancer. A topographical analysis on lymph node metastases. Eur J Cancer 2002;38:911-8.

35. Shihab OC, Brown G, Daniels IR, Heald RJ, Quirke P, Moran BJ. Patients with low rectal cancer treated by abdominoperineal excision have worse tumors and higher involved margin rates compared with patients treated by anterior resection. Dis Colon Rectum 2010;53:53-6. 


\section{Supplementary}

\section{Supplementary Appendix S1}

\section{Patient preparation for magnetic resonance imaging (MRI)}

Patients underwent rectal cleansing using $10 \mathrm{~mL}$ of glycerin enema to avoid possible misinterpretation due to residual stool, and $10 \mathrm{mg}$ of raceanisodamine hydrochloride was intramuscularly injected 20-30 minutes before MRI to reduce intestinal peristalsis or rectal spasm, except in patients who had contraindications, such as intracranial hypertension, serious heart disease, glaucoma, or prostatic hypertrophy. A total of $50 \mathrm{~mL}$ of ultrasound gel was inserted into the rectal vault of each patient prior to examination.

\section{MR image acquisition}

Oblique axial, sagittal, and coronal non-fat saturated $\mathrm{T}_{2}$-weighted fast-spin echo images were obtained orthogonal or parallel to the long axis of rectal cancer. Pelvic axial $\mathrm{T}_{1}$-weighted imaging, $\mathrm{T}_{2}$-weighted sequence with fat saturation, and diffusionweighted imaging were also performed to facilitate the detection of lymph nodes. A contrast-enhanced 3D $\mathrm{T}_{1}$-weighted gradient-echo sequence was acquired following intravenous administration of gadolinium-based contrast medium. Details of the protocols are listed in Table S1.

Table S1 Protocols for the magnetic resonance imaging sequences

\begin{tabular}{|c|c|c|c|c|c|c|c|c|c|}
\hline Protocol sequence & $\mathrm{TR}(\mathrm{ms})$ & $\mathrm{TE}(\mathrm{ms})$ & $\mathrm{FOV}(\mathrm{mm})$ & Matrix & Bandwidth (kHz) & NEX & ETL & Slice thickness (mm) & Intersection gap (mm) \\
\hline Sagittal T2WI & 4,800 & 115 & 240 & $256 \times 320$ & 41 & 4 & 21 & 4 & 0.4 \\
\hline Coronal T2WI & 4,800 & 115 & 240 & $256 \times 320$ & 41 & 4 & 21 & 4 & 0.4 \\
\hline T1WI & 560 & Min & 340 & $288 \times 224$ & 41 & 2 & 4 & 5 & 0.5 \\
\hline DWI $(b=0,1,000)$ & 2,300 & Min & 340 & $128 \times 160$ & 250 & 2 & NA & 5 & 0.5 \\
\hline CE-T1WI & 3.4 & 1.6 & 300 & $288 \times 224$ & 125 & 1 & NA & 3 & 0 \\
\hline
\end{tabular}

CE, contrast-enhanced; DWI, diffusion-weighted imaging; ETL, echo train length; FOV, field of view; FS, fat saturation; NA, not available; NEX, number of excitations; TE, echo time; TR, repetition time; T1WI, $\mathrm{T}_{1}$-weighted imaging; T2WI, $\mathrm{T}_{2}$-weighted imaging. 


\section{Supplementary Appendix $\mathbf{S 2}$}

\section{Details of imaging analyses for the primary cohort}

Oblique axial $\mathrm{T}_{2}$-weighted imaging (T2WI) was used as the main sequence for evaluation. When a lymph node (LN) could not be discriminated clearly, other sequences such as sagittal, coronal T2WI, or diffusion-weighted imaging were used for assistance.

\section{Nodal location}

Nodal location was classified into the following four subtypes according to the position of nodes and primary rectal cancers: (I) ipsilateral and at tumor height; (II) ipsilateral and outside tumor height; (III) contralateral and at tumor height; and (IV) contralateral and outside tumor height.

\section{Chemical shift effect (CSE)}

CSE at the edge of nodes was categorized into the following three subtypes based on the presence and aspect of CSE: (I) regular CSE; CSE at the LN border is complete and smooth; (II) irregular CSE; CSE at the LN border is irregular, incomplete, or interrupted; and (III) absent CSE; CSE at the LN border is absent.

\section{Borders}

The borders of nodes were classified as well or poorly defined. Well-defined border indicated that the border of the node was regular and smooth; poorly defined border indicated that the border of the node was indistinct, lobulated, or spiculated (Figure S1).

\section{Internal signal (IS) pattern and signal intensity (SI)}

The IS was categorized as homogeneous or heterogeneous (Figure S2). The SI of nodes was classified as hypointensity, isointensity, or hyperintensity on T2WI compared with that of the nearby rectal wall (Figure S3). The signal from the major areas of the node that were heterogeneous was evaluated as the nodal SI.

\section{Long- and short-axis diameters}

The long- and short-axis diameters of each LN were measured in millimeters, and the ratio of the short- to long-axis diameter was calculated.

\section{Minimum distance to rectal tumor or rectal wall}

If the rectal tumor and the ipsilateral node were at the same height, the minimum distance from the node to the outer border of the tumor was recorded. Otherwise, the minimum distance from the node to the rectal wall was measured and recorded on oblique axial T2WI. 

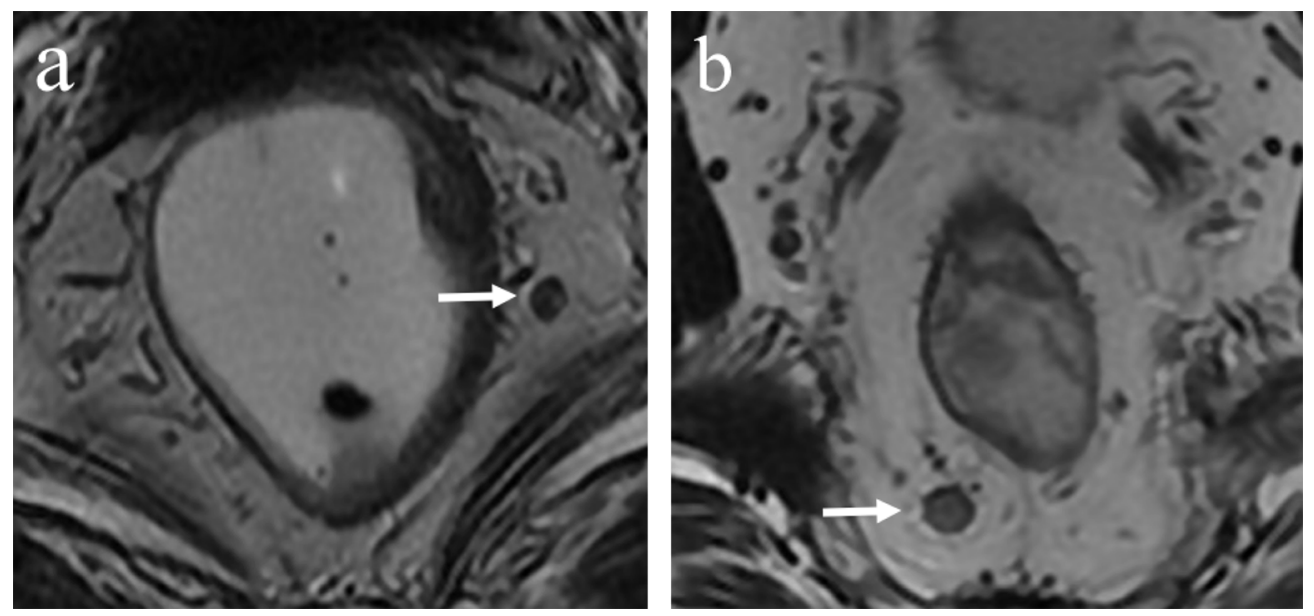

Figure S1 Borders of nodes on $\mathrm{T}_{2}$-weighted images (white arrows). (A) Well-defined border; (B) poorly defined border.
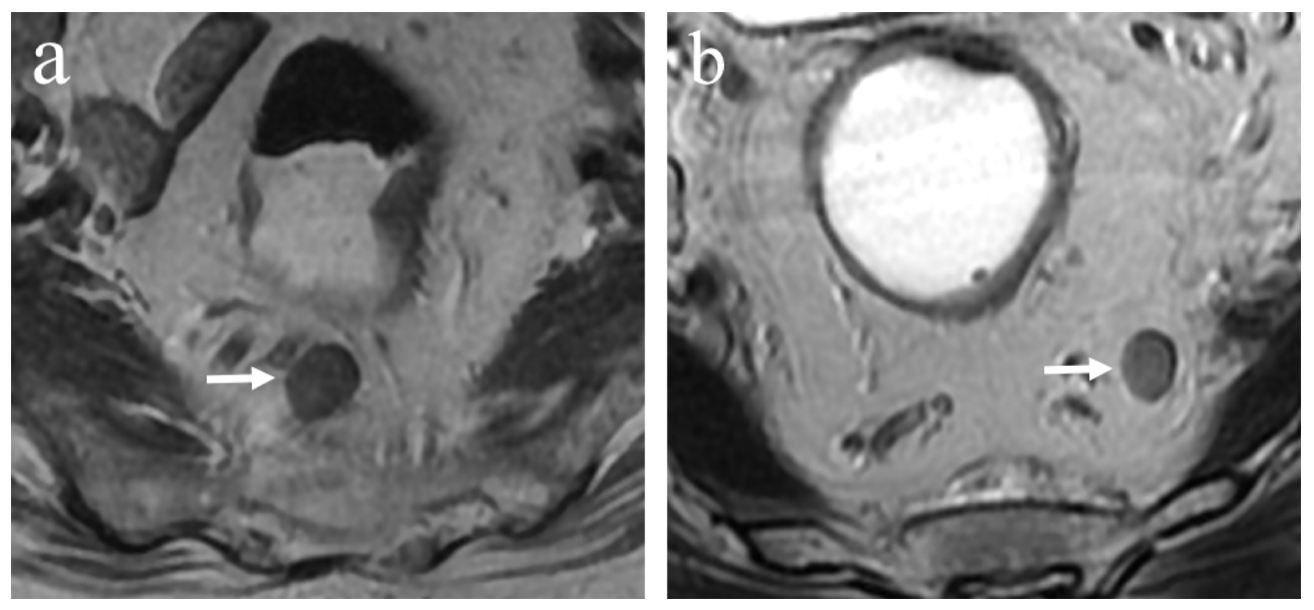

Figure S2 Internal signal (IS) pattern of nodes on $\mathrm{T}_{2}$-weighted images (white arrows). (A) Signal from the major areas of the node is heterogeneous; (B) signal from the major areas of the node is homogeneous.
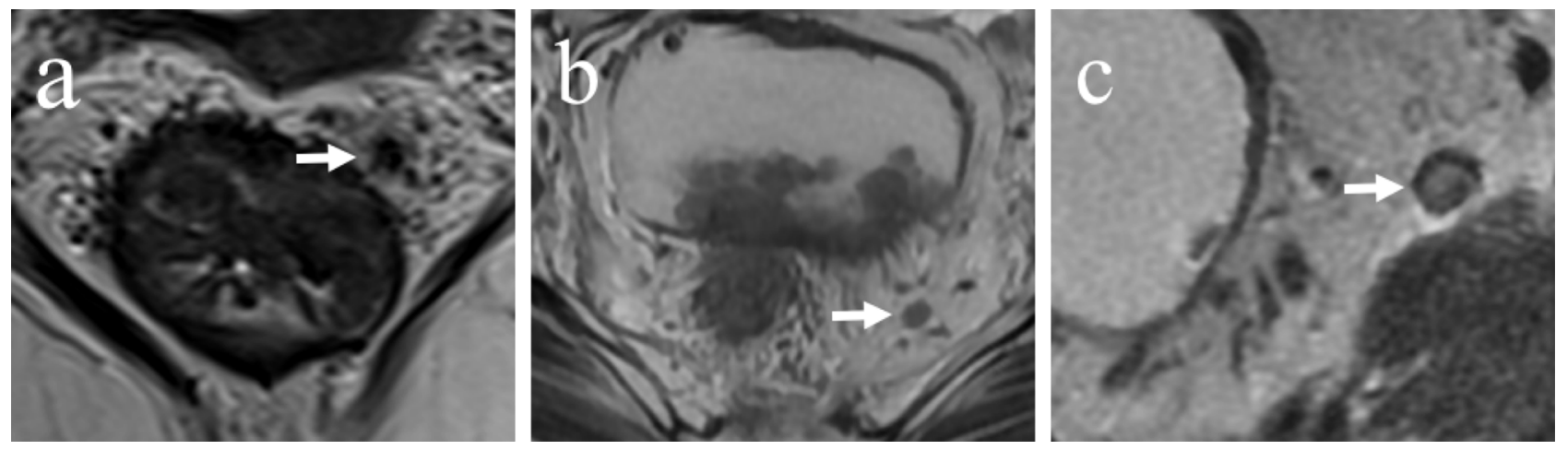

Figure S3 Three subtypes of signal intensity on $\mathrm{T}_{2}$-weighted images (white arrows). (A) Hypointensity; (B) isointensity; (C) hyperintensity. 